\title{
The application of signal processing techniques for real-time monitoring of a ship's dynamic stability via a ship's motion responses
}

\author{
Hossein Enshaei, Richard Birmingham
}

\begin{abstract}
Significant changes of stability at sea can lead to dangerous situations and eventually stability failure. Despite its importance, the current intact stability (IS) criteria do not evaluate the motion responses of a vessel. More recently, the International Maritime Organization (IMO) has identified phenomena in seaways responsible for stability failures. These phenomena can cause large roll angles and/or accelerations which can endanger ships due to critical stability situations in waves.
\end{abstract}

The measurement of waves whilst a ship is underway is a major challenge, but the ship's motions are a good reflection of the wave characteristics and can be captured. Signal processing techniques are used in the detection and estimation of a wave's influential parameters through the analysis of motion responses. Some variables of the system can be detected by spectral analysis of heave and pitch responses. These

key words: High roll, irregular wave, STFT, Spectrogram.

Hossein Enshaei $(\triangle)$, School of Marine Science and Technology, Newcastle University, UK. email: h.enshaei@ncl.ac.uk Tel:+44(0)191228113

Richard Birmingham, School of Marine Science and Technology, Newcastle University, UK. email: r.w.birmingham@ncl.ac.uk variables are the wave peak frequencies and associated magnitudes which can cause a high roll motion when in line with the roll natural frequency.

The instantaneous frequency (IF) present in the signal is revealed through spectral analysis of shorttime Fourier transforms (STFT) in less than a minute. The IF is a parameter of practical importance which can be used in decision making processes to avoid a high roll motion.

\section{Introduction}

Stability is the ability of a ship to float in an upright position and is one of the most important requirements for a ship's safe operation; insufficient stability can lead to the capsizing of the vessel and loss of life and property. It is essential to maintain adequate stability in all operational and loading conditions.

"When sailing in adverse weather conditions, a ship is likely to encounter various kinds of dangerous phenomena. They may lead to capsizing or severe roll motions causing damage to cargo, equipment and persons on board. The sensitivity of a ship to dangerous phenomena will depend on the actual stability parameters, hull geometry, ship size and ship speed. This implies that the vulnerability to dangerous responses, 
including capsizing and its probability of occurrence in a particular sea state may differ for each ship" $[1,8]$.

Current intact stability (IS) criteria are measures based on some parameters of a vessel and do not require the evaluation of the motion responses of a vessel. Significant changes in stability whilst at sea can lead to dangerous situations and eventually stability failure. Therefore, the Stability, Load Lines and Fishing Vessel Safety (SLF) Committee of the International Maritime Organization (IMO) has identified phenomena in seaways responsible for stability failures. These phenomena can cause large roll angles and/or accelerations which can endanger the ships due to critical stability situations in waves.

One of the major issues faced by the SLF committee in revising its IS Code in 2002 was the development of "performance-based", or "dynamics-based" criteria to predict stability failures. Performance oriented criteria, based on a physical model, is an option to address failure modes [10]. In contrast, parametric criteria are based on the measurement of a quantity which is related to a phenomenon. However, Belenky has defined "vulnerability" as the susceptibility of a ship's stability to the adverse effects of the environment and in particular, extreme motion behaviour [2]. He considers that it would be largely supportive if such criteria could be based on physical considerations and therefore be applicable to any type of vessel.

It is evident that the IMO rules are slowly migrating from a deterministic environment to a probabilistic one, also taking into account the presence of waves. It was emphasised in the IMO IS Code of 2002 that a range of sea conditions should be taken into account in order to develop an instrument for evaluating the effect of ship design parameters on ship safety in a seaway. A reliable, easily applicable method is essential to alleviate the seaway dangers and should avoid any possibility of manual computation. Operational stability is very complex; however, it is important to understand the links between the sea state and a ship's course.

\section{Aims and objectives}

Accurate calculation of the change of stability in waves presents certain challenges. "Some combinations of wave length and wave height under certain operation conditions may lead to dangerous situations for ships complying with the ISC" [8]. The motion behaviour of ships in an environment characterized by waves and wind varies for different types and scopes, which adds to the complexity of dynamic responses at sea.

The aim of this study is to avoid a reduction of stability for a long period of time or at high roll angles which increase the probability of capsizing. A ship's motion is of a time-varying nature and can be measured by means of sensors as discrete time signals. Therefore, signal processing techniques can be employed as a tool to analyse such signals for operational purposes.

The objective of this study is to demonstrate a suitable technique and the development of a programme using signal processing techniques as the basis of motion responses. The heave and pitch motions are in tune with irregular wave patterns and therefore their responses can be regarded as applicable tools to detect and estimate influential wave parameters. The programme could help operators to take corrective action to prevent a dangerous situation occurring and eventually stability failure. This is a reliable method, which can be utilised as a supplement to the current IMO recommendations [8] to avoid dangerous situations in adverse weather and sea conditions. 
This paper uses the short-time Fourier transform (STFT) technique to detect and demonstrate the identified wave parameters. The vulnerability of ships in different seas and operating conditions can be monitored to provide early warning of critical stability situations. This early warning can be achieved in approximately half a minute.

If the master is notified of a deteriorating situation, then he would be able to make operational decisions in ample time by changing course and/or speed. The master is the sole person responsible for taking decisions in adverse conditions and should be provided with sufficient information to justify the subsequent manoeuvring action.

\section{Waves and}

\section{measurements}

Waves are disturbances of water and transmit vast amounts of energy, therefore understanding their motions and characteristics are essential. The forces generated by waves can pose a significant threat to commercial shipping, thus wave measurement and their analysis is of prime importance for ensuring safety of operation. Simple waves are readily analyzed in their perfect regularity but they do not accurately depict the variability of ocean waves. The sea surface is composed of waves of varying heights and periods moving in differing directions. When the wind is blowing and the waves are growing in response, the seas tend to be confused and a wide range of heights and periods is observed. Swell is more regular, but is fundamentally irregular in nature, with some variability in both height and period.

Spectral analysis is a powerful and popular approach that exists for treating complex waves. Spectral analysis assumes that the sea state can be considered as a combination or superposition of a large number of regular sinusoidal wave components with different frequencies, heights, and directions. This is a very useful assumption in wave analysis since sea states are in fact composed of waves from a number of different sources, each with its own characteristic height, period, and direction of travel.

Mathematically, spectral analysis is based on the Fourier transform of the sea surface. The Fourier transform allows any continuous, zero-mean signal, like a time series record of the sea surface elevation, to be transformed into a summation of simple sine waves. These sine waves are the components of the sea state, each with a distinct height, frequency, and direction. In other words, the spectral analysis method determines the distribution of wave energy and average statistics for each wave frequency by converting the time series of the wave record into a wave spectrum. This is essentially a transformation from the timedomain to the frequency domain, and is accomplished most conveniently using a mathematical tool known as the fast Fourier transform (FFT).

The spectral approach indicates which frequencies have significant energy content, and can readily be plotted in a frequency vs. energy density graph, which can provide important information about a wave sample and the corresponding ocean conditions. All of the valuable information produced by spectral wave analysis is based on a timeseries record of sea surface elevations. In general, time series are analyzed over short periods, from 20 minutes to several hours which is too lengthy for online monitoring. Without a proper technique for recording and analysing the waves experienced by a ship the results obtained can only be related to potentially unreliable visual estimates of the sea state.

The techniques commonly used for wave measurement are as follows: 
Sea

This was perhaps the most common observation included in early tabulations of wave-heights.

\section{- $\quad$ Satellite Altimeters}

Satellite altimeters are now the most commonly used tool for wave measurements. Altimeter data are used to produce monthly mean maps of wave-heights and the variability of wave energy density in time and space.

\section{- $\quad$ Radar wave}

This maps the radar reflectivity of the sea surface and often shows wave-like features related to the real waves on the sea surface. There is not an exact one-to-one relationship between wave height and image density.

- Accelerometer Mounted on a Meteorological Buoy

This is a less common measurement although it is often used for measuring waves during short experiments whilst at sea.

\section{- $\quad$ Wave Gages}

Gauges may be mounted on platforms or on the seafloor in shallow water.

Accuracy, availability or delays in obtaining data for the analysis of time series are the main constraints to the implementation of the above methods for online monitoring of ship responses. However, considerable progress has now been made in the application of statistical methods to quantify the characteristics of waves on the sea surface, and these methods form one of the foundations of the modern theory of seakeeping [12].

It was in 1953 that it was first suggested that a ship could be treated as filter with an input not of electrical signals but instead waves [15]. The ship as a black box receives the waves as an input, which contains a number of different frequencies and components, and generates ship motions as the output (see figure 1).

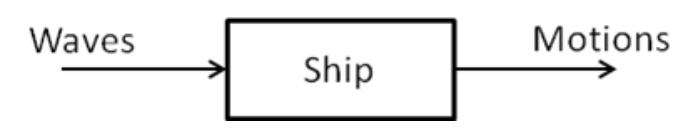

Fig. 1 The electronic filter analogy.

The measurement of waves whilst the ship is underway is a major challenge; however, taking the ship's parameters and loading conditions into consideration, then motion responses could be the best representative of the current sea state. Signal processing techniques are effective in the detection of a wave's influential parameters through the analysis of heave and pitch motion responses. These variables are the wave peak frequencies and associated magnitudes which can cause a high roll motion when in line with the roll natural frequency. STFT is used to analyse a recorded time series of a ship's motion responses for the specified period.

\section{$4 \quad$ Signal processing}

\section{methodology}

The majority of signals encountered in the real world change with time, and sea waves and swell can also be considered as signals with a time-dependent spectral content which are self-generated in nature. The processing of such signals can be used to form the basis of motion analysis of ships at sea.

From the mathematical perspective, a given signal can be represented in an infinite number of ways via different expansions. The Fourier transform has been the most commonly utilised tool to study a signal's frequency properties. The two most common approaches used to describe a signal's behaviour in terms of frequency, are the Fourier transform (linear) and the power spectrum (quadratic). However, based on the Fourier transform and the power spectrum alone, it is hard to determine whether or not a signal's frequency contents evolve over time. Although the phase characteristic of a 
frequency spectrum $(S(\omega))$ contains time information, it is difficult to establish the point-to-point relationship between a time series $(s(t))$ and a $S(\omega)$ based upon conventional Fourier analysis. Because the basis functions used in the classical Fourier analysis do not associate with any particular time instant, the resulting measurements do not explicitly reflect a signal's time-varying nature.

The introduction of time-frequency (TF) signal processing has led to the representation and characterization of a time-varying signal using a time-frequency distribution (TFD) [4]. TFD is a two dimensional function which simultaneously provides time and spectral information. Distribution of signal energy over the TF plane uncovers information not available in the time or frequency domain alone. This includes a number of components present in the signal, such as time duration, frequency bands, relative amplitudes, phase information and instantaneous frequency (IF) in the TF plane [14].

The typical aim of signal processing is to find a representation in which certain attributes of the signal are made explicit. Therefore, the central issues of signal processing are how to construct a set of the elementary functions $\left\{\psi_{n}\right\}_{n \in Z}$ and how to compute the corresponding dual functions $\left\{\hat{\psi}_{n}\right\}_{n \in Z}$. Either of these can then be used for analysis functions to compute the expansion coefficients or to transform. For good measurements, the set of analysis functions should be selected to give the easiest and finest measurements.

\subsection{Application of instantaneous}

\section{frequency}

The most important relationship in terms of joint time-frequency analysis is the relationship between a signal's time duration and the frequency bandwidth. Time duration and frequency bandwidth can be defined in several different ways and different definitions lead to different interpretations. One of the most common definitions is standard deviation, the concept used in the probability theorem to characterize the time duration and frequency bandwidth.

Parseval's formula for the signals $\mathrm{s}(\mathrm{t})$ and $\mathrm{h}(\mathrm{t})$ is defined as:

$$
\int s(\tau) h(\tau) d \tau=\frac{1}{2 \pi} \int S(\omega) H(\omega) d \omega
$$

If $s(t)=h(t)$ then:

$$
\int|s(t)|^{2} d t=\frac{1}{2 \pi} \int|S(\omega)|^{2} d \omega=E
$$

This implies that the Fourier transformation conserves energy.

The normalized functions $|s(t)|^{2} / E$ and $|S(\omega)|^{2} / 2 \pi E$ can be thought of as signal energy density functions in the time and frequency domains respectively. Given that, the moment concepts from the probability theory used to quantitatively characterize the signal's behaviour, the mean time and mean frequency computed from the first moment is:

$$
\langle t\rangle=\frac{1}{E} \int t|s(t)|^{2} d t
$$

and:

$$
\langle\omega\rangle=\frac{1}{2 \pi E} \int \omega|S(\omega)|^{2} d \omega
$$

To understand the relationship between the time representation and frequency behaviour, a derivative property needs to be taken into consideration. If $s(t)=A(t) \exp (j \varphi(t))$, then a new function 
$H(\omega)=\omega S(\omega)$ can be defined and substituted into equation (3) to obtain,

$$
\langle\omega\rangle=\frac{1}{2 \pi E} \int H(\omega) S^{*}(\omega) d \omega
$$

Where $A(t)$ and $\phi(t)$ are magnitude and phase respectively; both are real.

Applying Parseval's formula into equation (4) and replacing the $H(\omega)$ function with the derivative:

$$
h(t)=-j \frac{d}{d t} s(t)
$$

The following is obtained:

$$
\langle\omega\rangle=\frac{1}{E} \int-j \frac{d}{d t} s(t) s^{*}(t) d t
$$

By substitution of the $s(t)$ function and setting $t=0$, working out the derivative gives:

$$
\langle\omega\rangle=\int \varphi^{\prime}(t) \frac{|s(t)|^{2}}{E} d t
$$

The mean frequency in equation (7) is the weighted average of $\varphi^{\prime}(t)$ over the entire time domain. $\varphi^{\prime}(t)$ is defined as the mean IF. Boashash [3] makes clear that apart from differentiation of the phase and smoothing thereof, there are many well established methods for estimating the IF, for example adaptive frequency estimation techniques such as the phase locked loop (PLL), and extraction of the peak from time-varying spectral representations.

Processing the frequencies of the spectral peaks reveals unambiguous and accurate information about the IF's present in the signals (Shafi, 2009), which makes the IF a parameter of practical importance in situations such as a ship's motion applications. Similarly, the concept of variance can be used to measure the signal's energy spreading in time and frequency domains. If $2 \Delta_{t}$ and $2 \Delta_{\omega}$ are defined for the time duration and frequency bandwidth, then:

$$
\Delta_{t}^{2}=\frac{1}{E} \int(t-\langle t\rangle)^{2}|s(t)|^{2} d t=\int t^{2} \frac{|s(t)|^{2}}{E} d t-\langle t\rangle^{2}
$$

and:

$$
\begin{aligned}
\Delta_{\omega}^{2} & =\frac{1}{2 \pi} \int(\omega-\langle\omega\rangle)^{2} \frac{|s(\omega)|^{2}}{E} d \omega \\
& =\frac{1}{2 \pi} \int \omega^{2} \frac{|s(\omega)|^{2}}{E} d \omega-\langle\omega\rangle^{2}
\end{aligned}
$$

Equations (8) and (9) are the standard definitions of variance, and specify the signal's spreading with respect to mean time and mean frequency.

Frequency variance can also be expressed as a function of time [13], similar to mean frequency as discussed earlier:

$$
\begin{aligned}
\Delta_{\omega}^{2}= & \frac{1}{2 \pi} \int\left(\varphi^{\prime}(t)-\langle\omega\rangle\right)^{2} A^{2}(t) d t \\
& +\frac{1}{E} \int\left(A^{\prime}(t)\right)^{2} d t
\end{aligned}
$$

Equation (10) indicates that the frequency bandwidth can be determined by $\varphi^{\prime}(t)$ and the magnitude variation $A^{\prime}(t)$. To obtain a narrow band signal either the magnitude or phase could be smoothed.

\subsection{Alternative approaches in}

\section{signal processing}

The main focus is to determine a distribution that represents the signal's energy concentration simultaneously in time and frequency without blur and cross terms (CTs) in order that closely spaced components can be easily distinguished. TFD have been intensively studied over recent decades and theoretical properties together with application fields for existing methods have been developed and stretched. 
The spectrogram is the most widely used tool for the analysis of time-varying spectra and is currently the standard method for the study of non-stationary signals. It is expressed mathematically as the magnitude-square of the STFT. Theoretically, the spectrogram has severe drawbacks, since it provides biased estimators of the signal IF. Another approach for time varying signal analysis is a tool which was developed based upon the concept of scale rather than frequency [5] and is the squared modulus of the wavelet transform. In this technique, functions are obtained by scaling the centre frequency of the mother wavelet. Consequently, both transforms have similarities and can be viewed as a rotation in functional space to a different domain. Additionally, the basis functions are localized in frequency, making mathematical tools such as power spectra and scalegrams useful for identifying frequencies and calculating power distributions.

Although both wavelets and STFT can be used to study a signal's local behaviour, they are substantially different in many aspects. For STFT-based signal decomposition, virtually any function can be used as the window function, whereas, one main issue for wavelet analysis is how to generate a desired mother wavelet. The wavelet transform does not have a single set of basis functions like that of the Fourier transform, which utilizes only the sine and cosine functions. Instead, wavelet transforms have an infinite set of possible basis functions. Thus wavelet analysis provides immediate access to information that can be obscured by other time-frequency methods such as STFT. However, to apply wavelet analysis one needs a certain level of mathematical preparation.

A general approach to formulate time and frequency function is a distribution that will be highly concentrated along the IFs present in a signal without any CTs, thus displaying good resolution. The Wigner-Ville (WD) distribution is a form of TFD formulated by a multiplicative comparison of a signal with itself [16], expanded in different directions about each point in time. It is qualitatively different from a spectrogram, and produces the ideal energy concentration along the IF for linear frequency modulation.

However, the use of the WD in practical applications is limited [14] by the presence of CTs resulting from interactions between signal components. These CTs may lead to an incorrect visual interpretation of the signal's TF structure, and are also an interference to pattern recognition. Moreover, if the IF variations are nonlinear, then the WD cannot produce the ideal concentration.

These methods are known as quadratic TFDs because the representation is quadratic in signal. None can be effectively used in all possible applications because all suffer from one or more problems. However, STFT and its variations are simple and easy to manipulate and are still the primary and most commonly used methods for analysis of a time-varying signal.

\subsection{The short time Fourier}

\section{transform (STFT) approach}

One way to overcome the deficiency of providing both time and spectral information simultaneously as required by the regular Fourier transform, is to compare the signal with elementary functions [12] that are localized in time and frequency domains simultaneously, i.e.:

$$
\begin{aligned}
\operatorname{STFT}(t, \omega) & =\int s(\tau) \gamma_{t, \omega}(\tau) d \tau \\
& =\int s(\tau) \gamma_{t, \omega}(\tau-t) e^{-j \omega \tau} d \tau
\end{aligned}
$$

Equation (11) is a regular inner product and reflects the similarity between signal $\mathrm{s}(\mathrm{t})$ and the elementary function $\gamma(\tau-t) \exp (-j \omega t)$. The function $\gamma(t)$ usually has a short time duration and therefore it is named the window function. Equation (11) is called the STFT or windowed Fourier transform. 


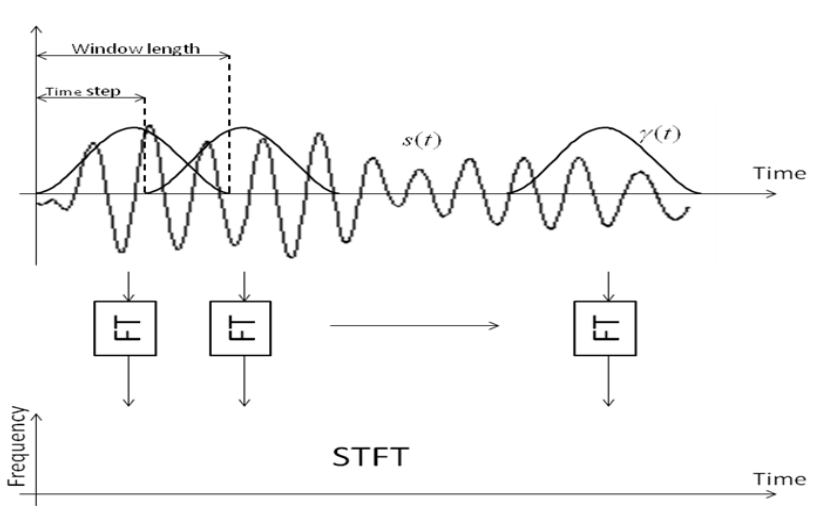

Fig. 2 Procedure to compute STFT.

The procedure for computing STFT can be interpreted in two different ways. As shown in figure 2, first the function $\gamma(t)$ is multiplied with signal $\mathrm{s}(\mathrm{t})$ before the Fourier transform is computed from the product $\mathrm{s}(\mathrm{t}) \gamma(t-\tau)$. Because the window function $\gamma(t)$ has a short time duration, the Fourier transform of $\mathrm{s}(\mathrm{t}) \gamma(t-\tau)$ reflects the signal's local frequency properties. By moving $\gamma(t)$ and repeating the same process, an approximation of how the signals frequency contents evolve over time can be obtained.

Alternatively, STFT could be explained from the concept of expansion. Signal $s(t)$ is compared with a set of elementary functions $\gamma(\tau-t) \exp (-j \omega t)$ that are concentrated in both time and frequency domains. If function $\gamma(t)$ is centred at $\mathrm{t}=0$ and its Fourier transform is centred at $\omega=0$, then the time duration and frequency bandwidth of $\gamma(t)$ are $\Delta_{t}$ and $\Delta_{\omega}$. STFT $(t, \omega)$ indicates a signal's behaviour in the vicinity of a reference point.

For a more accurate measurement of a signal at a particular time and frequency $(t, \omega), \Delta_{t}$ and $\Delta_{\omega}$ should be as narrow as possible. Due to the dependency of $\Delta_{\mathrm{t}}$ and $\Delta_{\omega}$ via the Fourier transform, if $\gamma(t)$ is chosen to have good time resolution (smaller $\Delta_{t}$ ), then its frequency resolution must be decreased (larger $\left.\Delta_{\omega}\right)$, or vice versa.
The latter approach has been chosen in this study to compute the STFT of a signal. A sliding window is used to divide the signal into several blocks of data. Next, a $N$-points FFT is applied to each block of data to obtain the frequency contents, where $\mathrm{N}$ is the frequency bins. The STFT aligns the centre of the first sliding window with the first sample of the signal and extends the beginning of the signal by adding zeros. The sliding window moves time step samples to the next block of data as shown in figure 2 .

The analysis function and synthesis function of continuous-time STFT have an identical form, therefore original time functions can easily be recovered. However, for a discrete signal the sampled STFT and recovery of an inverse form is not as simple. Although the concepts of exploring a time varying signal are quite similar (performing inner product operations), the different way of building elementary functions leads to very different outcomes. All linear transformations are achieved by comparing the analyzed signal with a set of prudently selected elementary functions.

\subsection{Accuracy of the presented} data

Numerous ideas have been developed to improve the concentration and resolution of TFDs for practical purposes. Theoretical properties and application fields for the large number of existing methods have now been well determined and are wide spread [7]. Although many quadratic TFDs have been proposed none can be effectively used in all possible applications.

The important feature of these methods is readability which corresponds to a good concentration of the signal components and no interference terms. This characteristic is necessary for visual interpretation of the outcomes and good discrimination between 
known patterns for non-stationary signal classification tasks. An ideal TFD function requires the following four properties [14]:

- $\quad$ High clarity which makes it easier to be analyzed. This requires high concentration and good resolution of the individual components for multi-component signals.

- Cross terms elimination which avoids confusion between noise and real components in a TFD for nonlinear TF structures and multi-component signals.

- Good mathematical properties which benefit its application. This requires that a TFD should satisfy the total energy constraint, marginal characteristics and positivity issue.

- Lower computational complexity means the time needed to represent a signal on a TF plane. Weak signal mitigation may increase computation complexity in some cases.

Previously, a spectrogram has been the most widely used tool for the analysis of time varying spectra [1] and it is currently the standard method for the study of nonstationary signals, and depicts a signal's energy distribution in the TF domain.

A spectrogram is expressed mathematically as the magnitude-square of the STFT of a signal, and is given by:

$$
S(t, \omega)=\left|\int x(\tau) h(t-\tau) e^{-i \omega \tau} d \tau\right|^{2}
$$

Where $x(t)$ is a signal and $h(t)$ is a window function.

However, this method of analysis suffers from a number of limitations:

- $\quad$ Time and frequency representations are related by the Fourier transformation, so that a signal's behaviour in the time domain and frequency domain are not independent. Therefore, the signal's time-duration and frequency bandwidth cannot be made arbitrarily small simultaneously.
- $\quad$ Presentation of data by a spectrogram provides bias estimation of the signal IF and should be taken into consideration when used as a measuring tool.

- $\quad$ All real measurements are disturbed by noise. This includes electronic noise, but can also include external events that affect the measured phenomenon, such as wind, wave, hull and engine vibrations, variations of temperature, variations of humidity, etc., depending on what is being measured and of the sensitivity of the device. Noise tends to spread evenly across the entire TF domain. When the characteristics of noise are known and are different from the signals, it is possible to filter it or to process the signal. Once the signal is identified, it is then possible to enhance the signal to noise ratio. In general, it is much easier to recognize a noisy signal in the TF domain than from either the time or frequency domain alone.

\section{Description of the}

\section{experimental set-up and}

\section{measurements}

In this study a Roll-on/Roll-off (Ro-Ro) model ship was used because of its vulnerability to greater stability losses which has been of great concern due to the large car deck spaces. Data provided from a previous study [6] is based on a range of significant wave heights and peak frequencies and were chosen to develop a particular short-term sea state with a Pierson Moskowitz (PM) sea spectrum.

The experiments involved systematic measurements of six degrees of freedom (6DOF) motion responses of a stationary model in an intact condition for three different headings in irregular seas. Qualisys Track Manager (QTM) with a ProRelex motion capture camera was used to track the motions and this provided a quick way to 
obtain accurate 6DOF information compared with traditional methods using motion sensors attached to a model. Waves were recorded by two pressure probes and data was recorded and analysed using the software LabVIEW [11]. Comparative observations of restorative motions were carried out for heave, pitch and roll responses.

The total number of recorded runs was 27, which consisted of three peak frequencies, with three significant wave heights and three heading angles, as shown in table 1.

Table1. Experimental wave conditions.

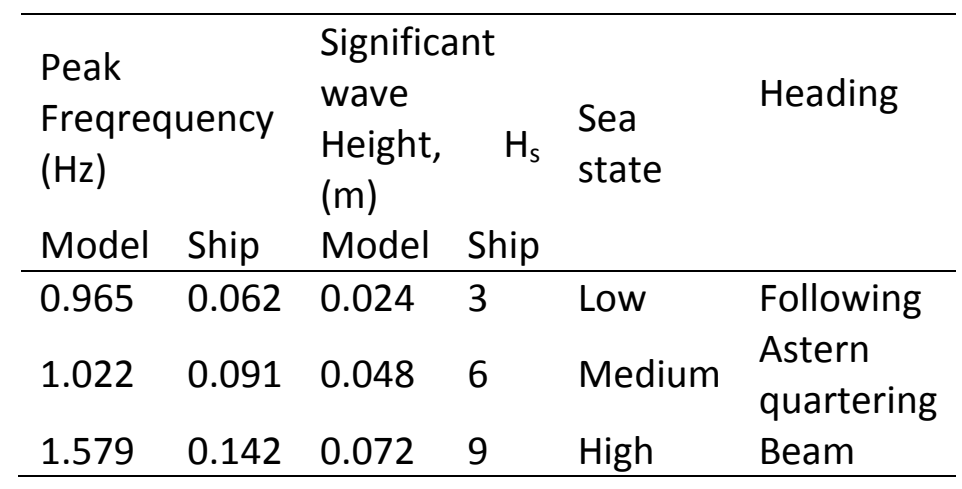

The wave conditions stated in table 1 were selected in order to maximise the possible test runs over a wide range of frequencies.

\section{Presentation and}

\section{discussion of the results}

A natural seaway can be decomposed into a sum of partial sinusoidal waves, each having a relatively small steepness, even for a severe sea. Therefore, the spectral approach with a sum of partial waves constitutes a valid representation for a random sea. Seaway spectra are narrow banded [9] and the total energy is concentrated at a narrow frequency range.
The discrete time varying data of the motion responses and incoming waves were analysed through the power spectral tool [11]. Figure 3 shows one of the test scenarios which demonstrates how the power spectral density (PSD) of an irregular wave, heave, pitch and roll motion is distributed over a range of frequencies during an 8 minute period.

A variety of methods were used to assess the signal for comparison purposes and figure 4 presents the wavelet analysis of the same scenarios as shown in figure 3. Wavelet analysis can be useful for identifying frequencies and calculating power distributions but in this case the extent of the information obtained provided little extra detail over that resulting from the PSD.

It was apparent that heave and pitch motions are strongly coupled and their responses have similar patterns. It is interesting to note that the peak frequency of both motions is very close to the wave peak frequency, whilst the roll motion tends to remain close to its natural frequency. This condition is more or less maintained in every sea condition and at every heading angle. These findings suggest that both heave and pitch motion responses are a good reflection of waves and can be used to determine present seaway peak frequency, and the outcome can be employed to predict a ship's roll behaviour.

The energy of a seaway changes gradually and can be considered to be constant for short periods of time. However, the duration of the above analysis may not be sufficiently short to provide ample time for decision making. Therefore a fast and reliable method is required for online monitoring of dynamic ship responses. 


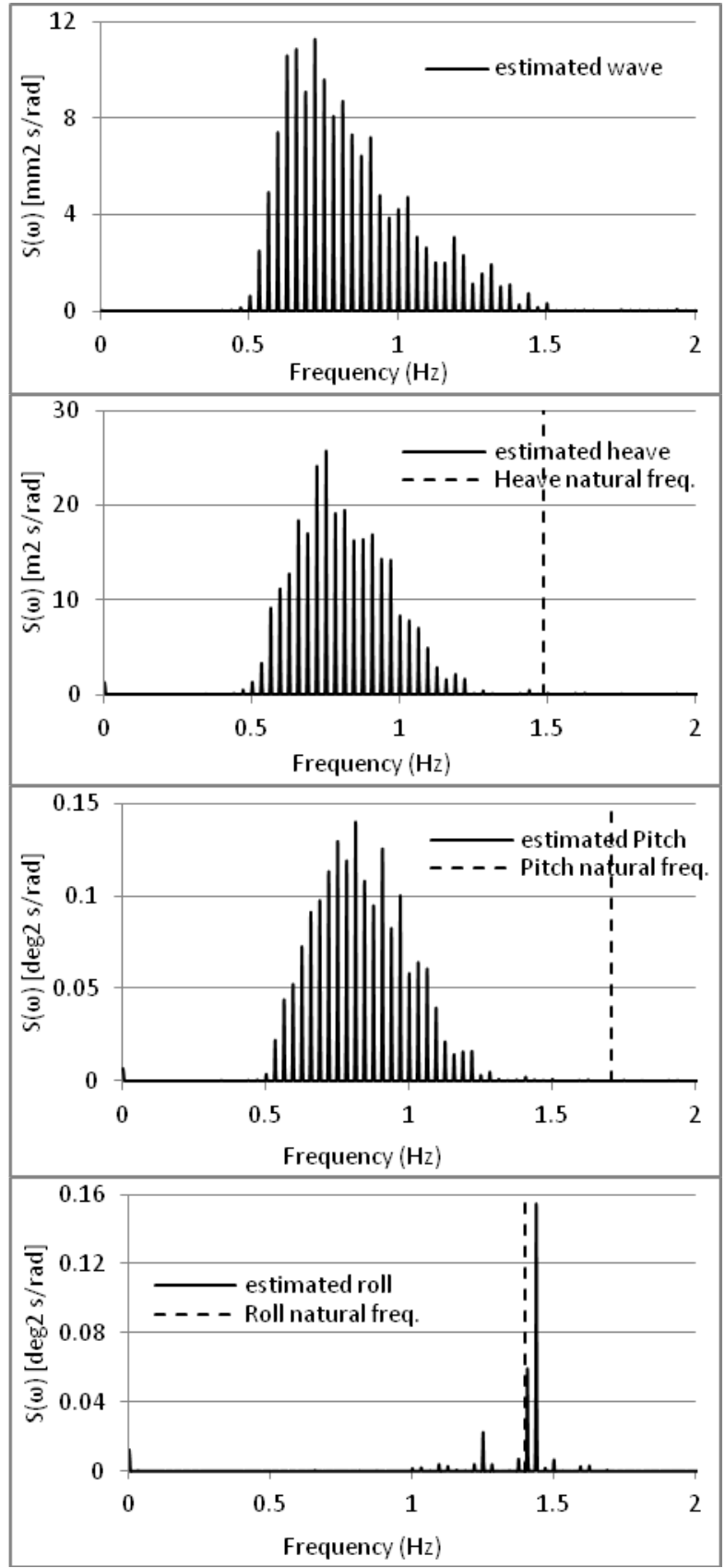

Fig. 3 Power spectral analysis of a model ship over an 8 minute period. a) irregular wave with peak frequency of $0.695(\mathrm{~Hz})$ and significant wave height $0.024(\mathrm{~m}), \mathrm{b})$ heave response motion, c) pitch response motion and $d$ ) roll response motion.

\subsection{Heave and pitch sensitivity to} irregular sea waves

The results obtained from the analysis of 27 test conditions indicate that the peak

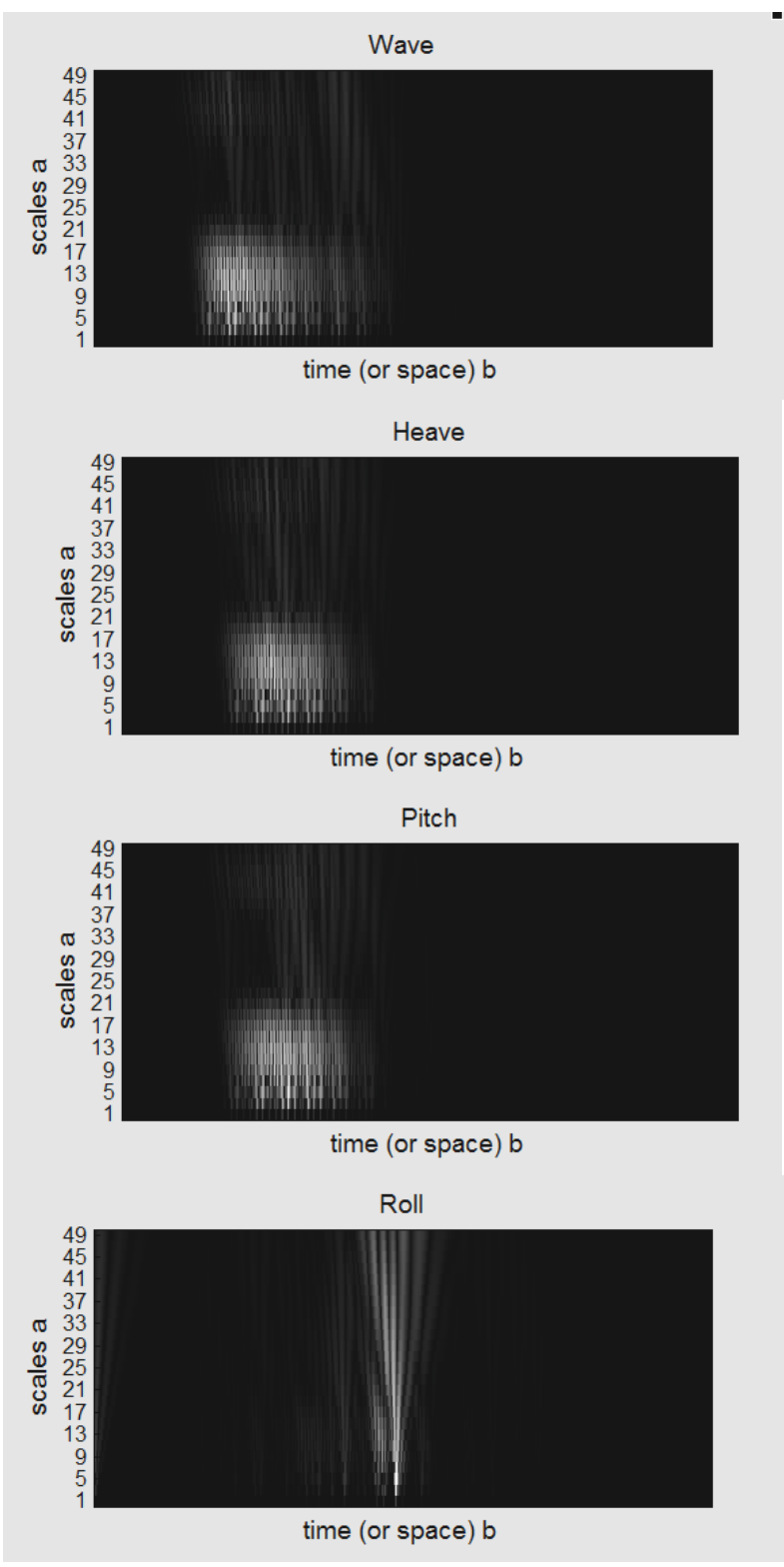

Fig. 4 Scalegram analysis of the same signals as presented in figure 3 .

frequency of heave motion and pitch motion track that of wave peak frequencies for every sea state, including significant wave heights and wave directions [6]. Figure 5 represents the motion responses of the ship; the $x$-axis describes the sea conditions (defined as 'low', 
'medium' and 'high' for the three different peak frequencies utilised in generating the wave spectrum) and the $y$-axis is the estimated frequency peak $(\mathrm{Hz})$. The results are presented for the three values of $\mathrm{H}_{\mathrm{S}}$ and are defined as 'small', 'medium' and 'large'.

In a following sea the coupling effects of heave and pitch motions are obvious at every $\mathrm{H}_{\mathrm{s}}$ value tested and closely follow the peak frequency of waves in different sea conditions.

The results in a quartering sea are interesting because the coupling effects of heave and pitch motions are similarly maintained throughout the change in heading angles as shown in figure 5 . In a beam sea the strong couplings amongst motion responses are dominated by roll and heave motions around the roll natural frequency. The pitch motion response tracks the wave peak frequency but is less sensitive at the low sea condition.

It was observed that the changes in heave and pitch magnitudes are in line with changes in wave magnitudes. However, the roll motion maintains its highest oscillation around the natural frequency in all sea conditions, regardless of the heading angle. Therefore roll is magnified when the peak frequency of the wave approaches the natural roll frequency; alteration of course away from a beam sea improves the roll motion.

\subsection{Roll sensitivity to irregular}

\section{sea waves}

The results obtained from figure 3 indicate that although heave and pitch motion identifies the wave pattern, the roll motion

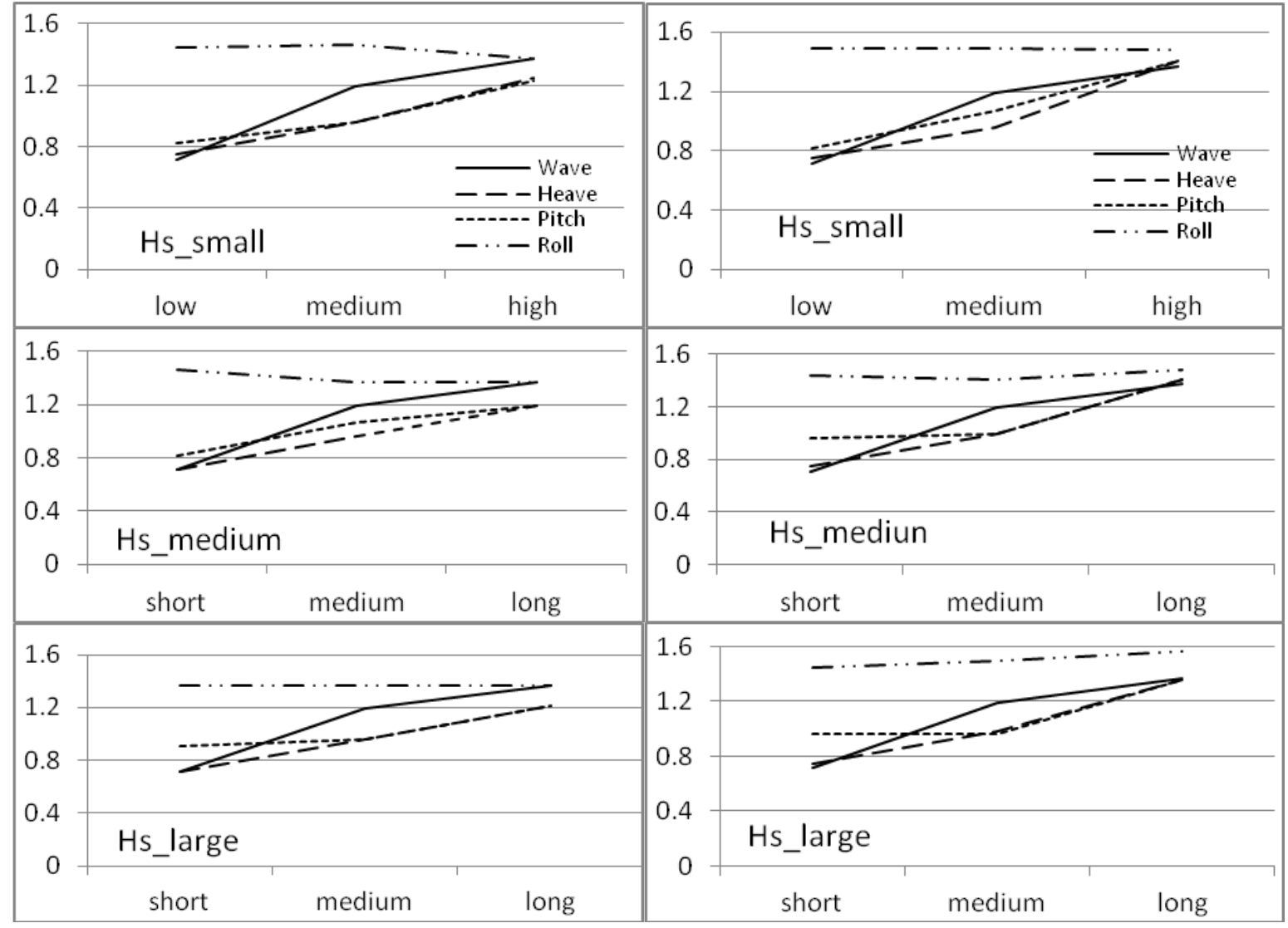

Fig. 5 Comparison of peak frequencies for each sea condition tested. Left: following sea, Right: astern quartering sea. 
follows its natural frequency. Figure 6 presents the sensitivity of the roll motion with respect to the wave peak frequency for different wave directions and significant wave heights. The results obtained show that the roll motion is magnified whilst the irregular wave peak frequency is in close proximity to the roll natural frequency. It is also apparent that there is a direct relationship between roll intensity and significant wave height for all sea conditions.

The inconsistent roll response in a following sea could be due to parametric rolling where both heave and pitch peak frequencies are about half of the roll natural frequency. Further work needs to be done with regards to this aspect of dynamic behaviour.

\subsection{Detection of wave influential}

\section{parameters}

For online monitoring of a ship's dynamic stability, a relatively reliable and fast method is required to detect wave peak frequencies and associated magnitudes. By referring to figure 3 it is evident that a timeframe of eight minutes is rather long and a ship could have been lost in the case of a critical situation. The STFT approach was chosen to analyse the recorded data. These data are from incoming waves as well as heave, pitch and roll motions in the form of a discrete time varying signal. STFT has a number of attractive features which can detect signal characteristics that are not obvious in the time or frequency domain alone. Detection and estimation of peak frequency and associated amplitude is achieved in less than a minute.

Spectrograms of each motion response are shown in figures 7 after computing the STFT of the signal. The STFT spectrogram is calculated as the magnitude square of the elements in STFT. Because the FFT returns symmetrical results, the spectrogram shows only the left half of the STFT. Note: The top left window of the spectrogram contains the intensity graph. The spectrogram returns the quadratic time-frequency representation of a signal and each row corresponds to the instantaneous power spectrum at a certain time. The lower window depicts the sampled time signal and the right window the corresponding frequencies of the sampled signal obtained by FFT. In figures 7 the detected and estimated peak frequencies and magnitudes of heave, pitch and roll responses are illustrated for a sampled signal of $20.5 \mathrm{sec}$ duration.

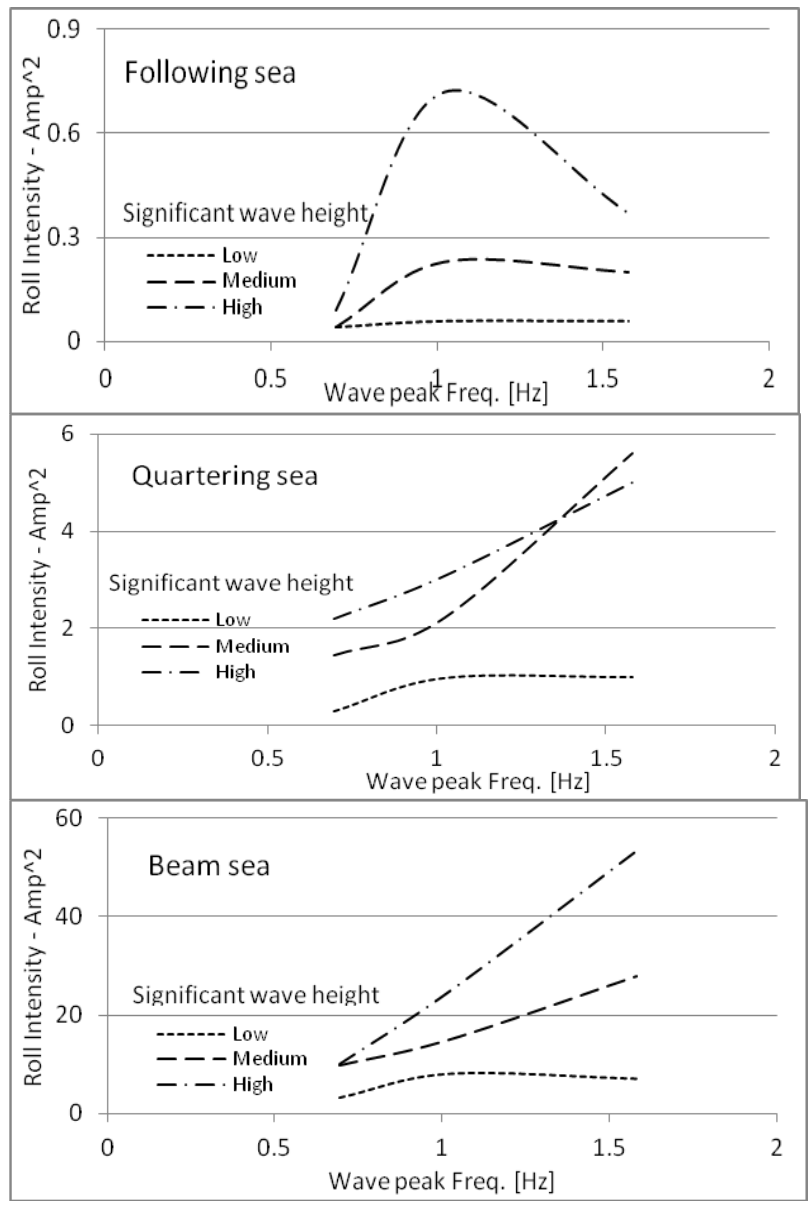

Fig. 6 Roll Intensity is a function of significant wave height and proximity of wave peak frequency to roll natural frequency.

\subsection{Effect of window and} sampling on the quality of detection

An important feature of a spectrogram is the readability which corresponds to a good 
concentration of the signal components. The type of window and sampling size can influence the visual interpretation which is necessary for good discrimination between known patterns.

\section{Window effect}

A window function is a mathematical function that is zero-valued outside of a chosen interval. The window functions used are nonnegative smooth "bell-shaped" curves, though rectangle and triangle functions and other functions are also sometimes used. Different window types employed in the detection of TF roll response are shown in figure 8. There is little difference in the detected magnitudes whereas the differences are significant for peak frequencies.

\section{Sampling size}

A comparison of three sample sizes is shown in figure 9, indicating that the quality of detection is affected considerably. It is apparent that variation in magnitude is significant and that the result is also influenced by the sampling rate which is $100 \mathrm{~Hz}$ in this scenario.

\section{Window length}

This shapes the time resolution and the frequency resolution of the STFT. A narrow window results in fine time but coarse frequency resolution because of the short time duration and wide frequency bandwidth. However, a wide window results in fine frequency but coarse time resolution. Therefore the window length should be calibrated for optimum concentration and obviously better resolution. The energy density distribution of a roll motion response is shown in figure 10 for different window lengths.

\section{Frequency bin}

This specifies the FFT size of the STFT. The frequency bin defines the number of bins which directly affect the resolution. It should be adjusted for optimum concentration and improved resolution. The energy density distribution of a roll motion response is shown in figure 13 for different frequency bins.
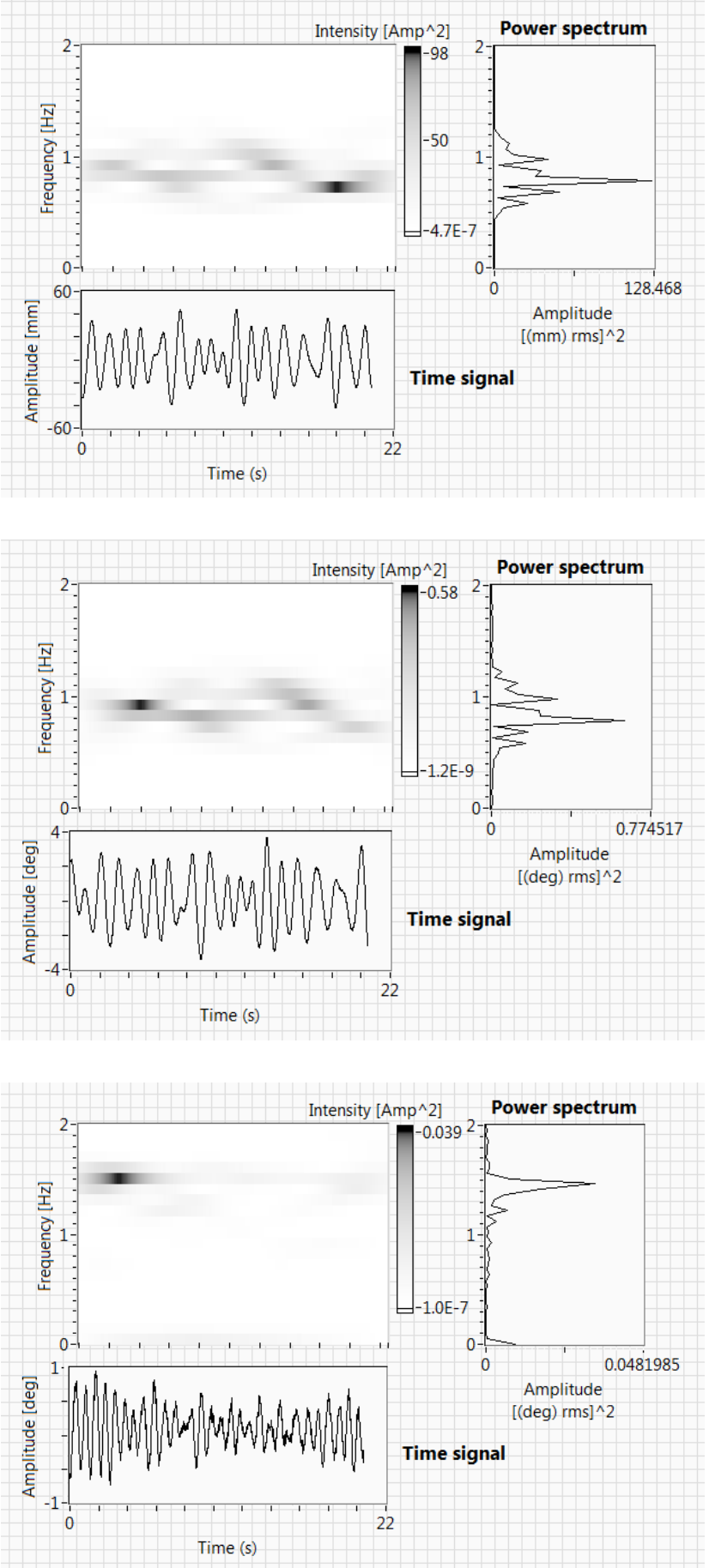

Fig. 7 TF analysis of a) heave motion, b) pitch motion and c) roll motion. The spectrogram shows energy density distribution as well as peak frequency. 


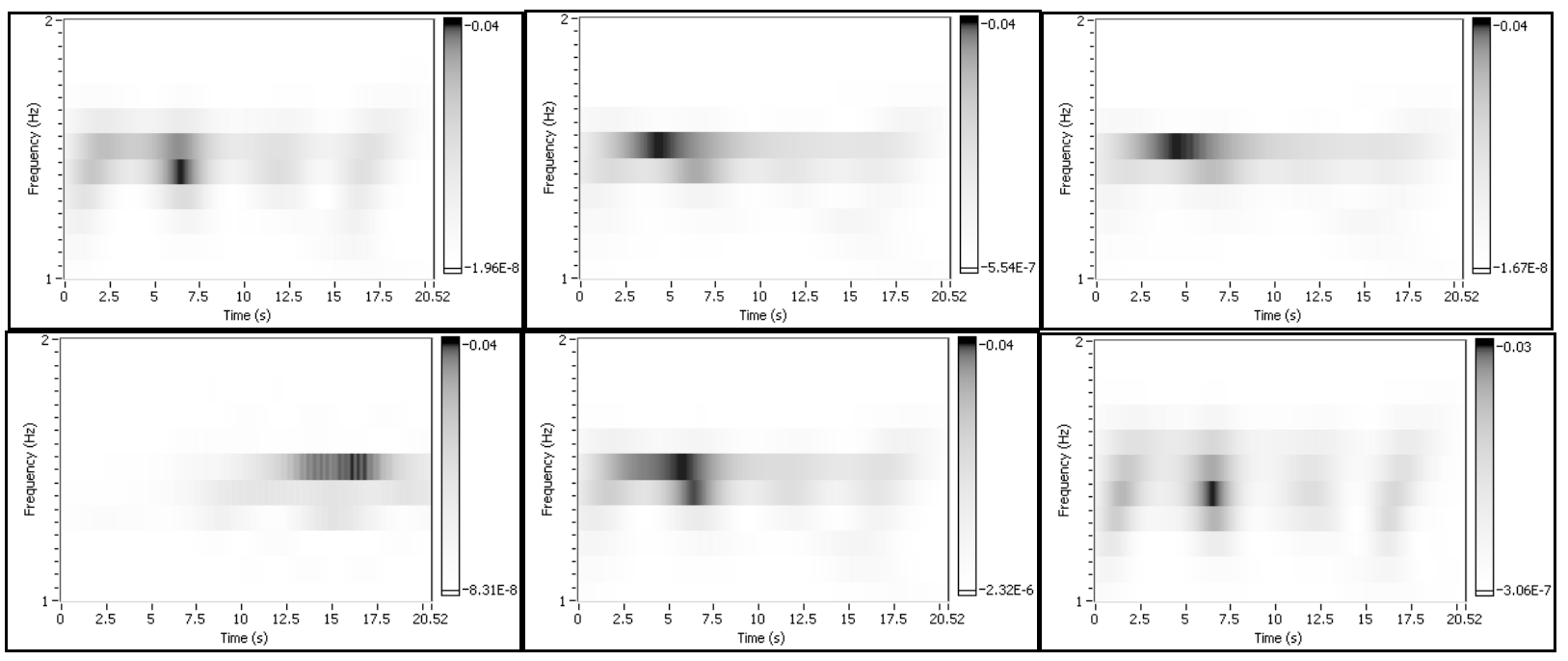

Fig. 8 Roll motion responses to an irregular wave with a peak frequency of $0.695 \mathrm{~Hz}$ and a significant wave height (Hs) of $0.024 \mathrm{~m}$ in a following sea. From top left, a) Gaussian, b) Hanning, c) Hamming, d) Rectangle, e) Blackman-Harris, f) Flat top.

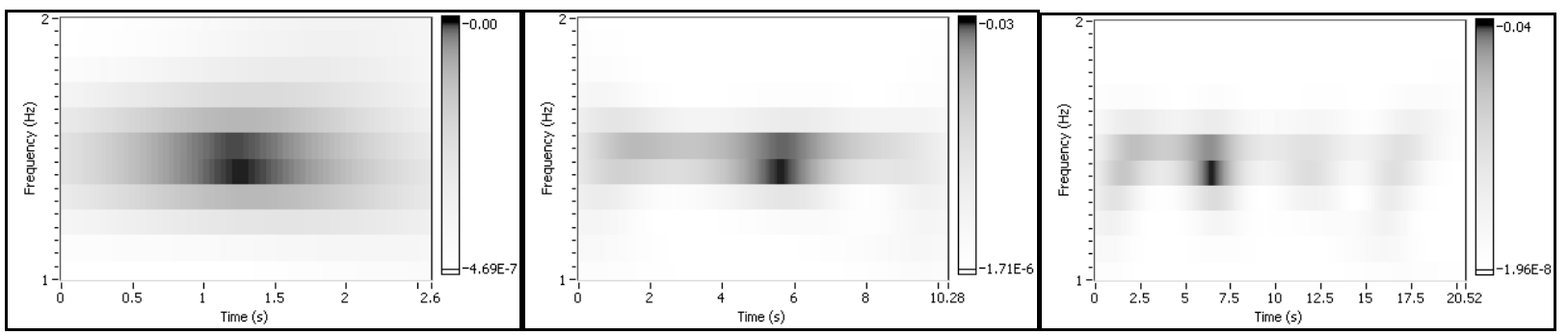

Fig. 9 Effect of sample size on quality of detection. From left a) $256 \mathrm{~Hz}$, b) $1024 \mathrm{~Hz}$ and c) $2048 \mathrm{~Hz}$.

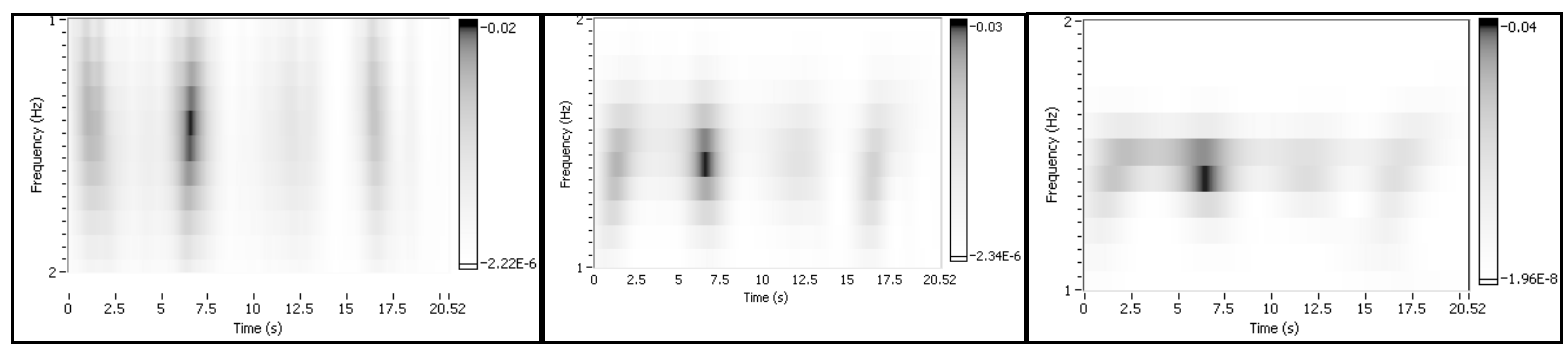

Fig. 10 Detection of a roll motion signal for different window lengths. From left a) $256 \mathrm{~Hz}$, b) $512 \mathrm{~Hz}$ and c) $1024 \mathrm{~Hz}$

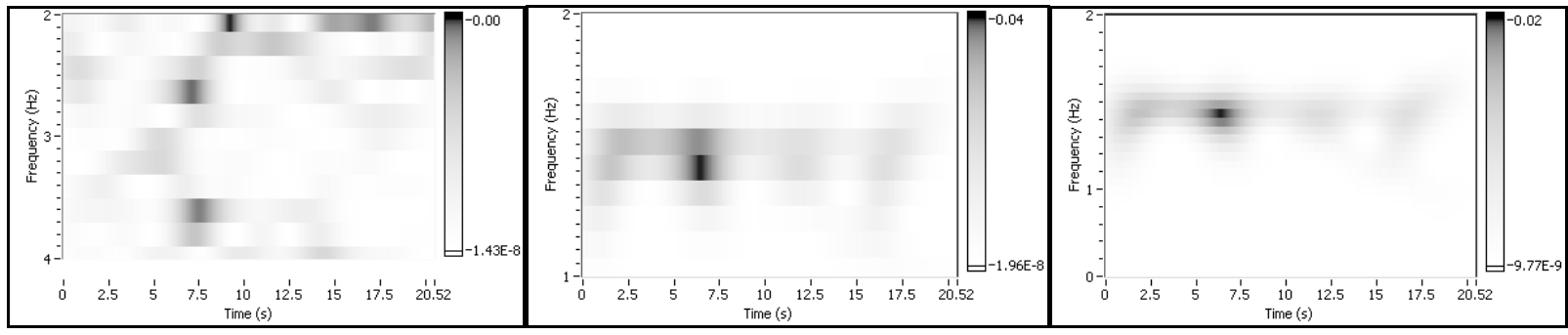

Fig. 11 Effect of frequency bins on the detection of a roll motion signal. From left a) 256, b) 1024 and c) 2048 


\section{Conclusions}

This study is based on the spectral analysis of a motion response test of a model of a Ro-Ro vessel in irregular waves. This has produced results which corroborate the findings of previous work. However, the main objective was to explore the signal processing technique to detect and estimate the peak frequency and associated magnitude of encountered waves. The following conclusions can be drawn from this study:

- $\quad$ The evidence from this study suggests that STFT is a suitable method for the detection and estimation of a time varying signal of motion responses.

- $\quad$ The IF and power magnitude of heave, pitch and roll motions could be obtained through this signal processing technique.

- $\quad$ One of the most significant findings to emerge from this study is that the detection of peak frequencies and associated magnitudes of motions could be achieved in less than a minute.

- $\quad$ The study has shown that heave and pitch motions can be used to identify the encountered wave pattern and therefore enable the wave's peak frequency and magnitude to be predicted.

- High roll motion can be avoided by monitoring the roll natural frequency and ensuring it is not close to the wave peak frequency. Therefore online monitoring of dynamic stability can be achieved.

If this debate is to be progressed, a better understanding of different ships' hull responses needs to be developed. More broadly, research is also needed to consider the effect of length, displacement and speed.

\section{REFERENCES}

1. Altes, R. A.: Detection, estimation and classification with spectrograms. Journal of the Acoustical Society of America, 67, (4), pp. 1232-1246 (1980).

2. Belenky, V., Ottodekat, J. et al.: Toward performance-based criteria for intact stability. Marine Technology, 45, (2), pp. 101-123 (2008).

3. Boashash, B.: Estimating and Interpreting the Instantaneous frequency of a signal-part II. Algorithms and Applications. Proceedings of the IEEE, 80, (4) (1992).

4. Boashash, B.: Time-Frequency signal analysis and processing. Prentice-Hall, NJ, USA (2003).

5. Daubechies, I.: The wavelet transform, timefrequency localization and signal analysis. IEEE Transactions on Information Theory, 36, (5), pp. 9611005 (1990).

6. Enshaei, h., Birmingham, R.: Identification of influential parameters in a ship's motion responses: a route to monitoring dynamic stability. Trans RINA, Vol 154, (Part A1), pp. Intl J Maritime Eng (2012).

7. Hlawatsch, F. a. B.-B., G. F.: Linear and quadratic time-frequency signal representations. IEEE Signal Processing Magazine, 9, (No. 2), pp. 21-67 (1992).

8. IMO.: Revised guidance to the master for avoiding dangerous situations in adverse weather and sea conditions. MSC.1/Circ.1228 (2007).

9. Kobylinski, K. L., Kastner. S.: Stability and safety of ships Vol I: Regulation and Operation. Elsevier Ocean Engineering book series, 9 (2003). 
10. Kobylinski, L. K.: Proposed approach to stability requirements based on goal determination and risk analysis. Maritime university of Szczecin, 13(85), (33-39) (2008).

11. LabVIEW: Graphical programming for instrumentation (2009).

12. Lloyd, A. R. J. M.: Seakeeping. Ellis Horwod, Chichester, Sussex, UK (1998).

13. Qian, S. a. C., D.: Joint Time-frequency Analysis. Prentice-Hall, NJ, USA (1996).
14. Shafi, I., Ahmad, J., Ismail Shah, S., Kashif, F.M.: Techniques to Obtain Good Resolution and Concentrated Time-Frequency Distributions: A Review. EURASIP Journal on Advances in Signal Processing, 2009, (Article ID 673539), pp. 43 (2009).

15. St Denis, M. P., W.J.: On the motions of ships in confused seas. TSNAME (1953).

16. Stankovic, L. J. a. S. S.: Analysis of instantaneous frequency representation using timefrequency distributions-generalized Wigner distribution. IEEE Transactions on signal processing, 43, (No. 2), pp. 549-552 (1995). 